\title{
Skin microbiota dynamics following $B$. subtilis formulation challenge: an in vivo study in mice
}

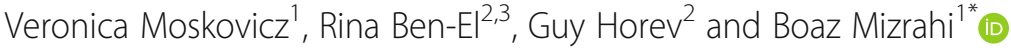

\begin{abstract}
Background: Modulating the microbiota is a leading-edge strategy for the restoration and maintenance of a healthy, balanced environment. The use of health-promoting bacteria has demonstrated some potential benefits as an alternative for skin microbiota intervention. Here, we investigate the manipulation of mice skin microbiota using B. subtilis incorporated into a supportive Pluronic F-127 hydrogel formulation. The formula plays an important role in delivering the bacteria to the desired action site.

Results: The B. subtilis challenge induced a shift in the composition and abundance of the skin microbiota. Containment of B. subtilis in the Pluronic F-127 hydrogel accelerated bacterial modulation compared with free $B$. subtilis. The abundance of both Staphylococcus and Corynebacterium spp. was altered as a result of the live bacterial intervention: the abundance of Corynebacterium increased while that of Staphylococcus decreased. Four days after last application of the B. subtilis formulation, B. subtilis counts returned to its initial level.

Conclusions: B. subtilis intervention can induce a shift in the skin microbiota, influencing the abundance of commensal, beneficial, and pathogenic bacteria. Containment of B. subtilis in Pluronic hydrogel accelerates the microbial alteration, probably by facilitating bacterial attachment and supporting continuous growth. Our results reveal the ability of $B$. subtilis in Pluronic to modulate the skin microbiota composition, suggesting that the formulation holds therapeutic potential for skin disease treatment.
\end{abstract}

Keywords: Bacillus subtilis, Live bacterial delivery, Pluronic F-127, Microbiota, Skin

\section{Background}

The skin, our largest organ, serves as the principal mechanical and biological protective barrier for the human body. Being the organ most exposed to the environment, the skin is colonized by a diverse collection of microorganisms that constitute the microbiota [1]. By communicating with epithelial cells and the immune system, the microbiota plays an important role in protecting the skin from potential damages posed by pathogenic microorganisms [2, 3]. Moreover, findings of the

\footnotetext{
* Correspondence: bmizrahi@technion.ac.il

${ }^{1}$ Faculty of Biotechnology and Food Engineering, Technion - Israel Institute of Technology, 3200003 Haifa, Israel

Full list of author information is available at the end of the article
}

Human Microbiome Project have revealed that many of the resident microorganisms are harmless (commensals) or have a positive influence on human skin health and well-being (mutualists) [4, 5]. However, when homeostasis is disrupted, the microbiota enters into a state of microbial imbalance, termed dysbiosis, which can lead to dermal immune dysregulation [6,7]. Skin microbiota dysbiosis has been associated with skin diseases including atopic dermatitis [8], acne [9], and vitiligo [10].

Several strategies for the manipulation of the skin microbiota have been suggested, including hygiene products [11], antibiotics [12, 13], and prebiotics [14]. Extrinsic factors, ranging from cosmetics to the environment and antibacterial agents, as forces that impact the 
human skin microbiome and well-being, were recently reviewed [15]. These methods, however, often suffer from poor efficacy and lack of selectivity towards the pathogenic bacteria [16]. Antibiotics, for example, have been associated with long-term bacterial imbalances, lasting up to several years and leading to an increased incidence of skin lesions [17, 18]. Antibiotic treatment has been showed to enhance depigmentation in vitiligoaffected skin [13] and to delay wound healing [19]. These drawbacks are driving the exploration of new microbiota intervention alternatives.

The use of health-promoting bacteria has shown promising results in the restoration of a healthy microbiota, for example by promoting the growth of beneficial microbes [20-23]. Bacillus subtilis has potential as a microbiota-modulating agent since it can efficiently outcompete important human pathogens such as E. coli and S. aureus $[24,25]$, probably through the production and secretion of potent antimicrobial agents [25] while displaying a non-pathogenic profile [24, 26, 27]. Moreover, B. subtilis efficiently outcompetes important human pathogens such as E. coli and S. aureus in vitro [24, 25], probably through the production and secretion of potent antimicrobial agents [25]. However, before this knowledge can be translated into therapeutic applications, additional groundwork is required and well-controlled in vivo studies to assess microbiota dynamics as well as safety and efficacy aspects of living bacteria interventions must be performed. The aim of this study is, therefore, to explore the experimental manipulation of the skin microbiota using a B. subtilis formulation incorporated into a supportive Pluronic F-127 hydrogel delivery matrix. Pluronic F-127 Poly (ethylene oxide)-poly(propylene oxide)-poly(ethylene oxide)) was selected as the main matrix due to its lower critical solution temperature, around body temperature, since it allows bacillus to grow inside the formula and prolonged the retention of $\mathrm{B}$. subtilis on the skin without compromising bacteria's ability to produce and secret its wide range of potent antimicrobial agents [28]. The microbiota of healthy skin was mapped before, during, and after the administration of a living $B$. subtilis formulation to monitor its dynamics. We hypothesized that a $B$. subtilis microbiota intervention will result in a microbial shift that will be limited to the treatment course, since human skin microbiota is relatively stable in terms of its microbial population $[29,30]$. One possible explanation for skin microbiome stability may well be that transient bacteria do not tend to establish themselves permanently on the skin, but rather persist only for hours to days [31].

\section{Results}

The effects of challenging the healthy mouse ear skin microbiota with a B. subtilis formulation were studied using the ear skin of laboratory mice. This procedure has been used as a model system for human skin sites in terms of morphology and microbiota and has been successful in assessing host-microbe interactions [32, 33]. Twenty-four 8-week old C57BL/6 female mice were randomly assigned to one of four groups: Pluronic hydrogel containing B. subtillis, B. subtillis (in LB medium), plain Pluronic hydrogel, and a no-treatment control group (Fig. 1A). Each group was administered with the corresponding formulation twice a day for seven days. The effects of the various treatments were analyzed by determining the bacterial composition of the skin before the first application (day 0 ) and on days $2,4,8,11$, and 14 (Fig. 1B). Of note, all animals did not show any sign of discomfort or changes in general behavior.

After skin sampling, genomic DNA was extracted and the V3-V4 hypervariable regions of $16 \mathrm{~S}$ rRNA gene were amplified and sequenced using the Illumina technology. A total of 29.5 million high-quality $16 \mathrm{~S}$ rRNA gene sequences were obtained, each containing between 0.36 and $1.5 \%$ of the data. Noise was removed according to the Remove Unwanted Variation (RUV) strategy [34], using the untreated control group and day 0 samples (before treatment) for normalization (Supplementary Table 1).

The dynamics of the abundance of B. subtilis, in particular, and of the Bacillus genus, in general, before, during, and after administration of B. subtilis formulations was assessed (Fig. 2A and B) and compared with the average abundances of B. subtilis and Bacillus (dashed lines), respectively. Indeed, plain Pluronic hydrogel did not impact the abundance of $B$. subtilis, as no B. subtilis was present in untreated samples (Fig. 2A). B. subtilis in Pluronic, conversely, had the highest influence on $B$. subtilis counts on days 2 and 4, which were significantly higher than for the B. subtilis treatment ( $\log _{2}$ differential expression 6.6 and 3.6 respectively, padj $<0.05$ ). Nevertheless, four days after the last administration, all groups presented counts that were similar to that of the untreated group. For the Bacillus genus (Fig. 2B), counts were significantly higher for all groups compared with the untreated control group: The group that received $B$. subtilis showed a 2-fold increase while the two groups that received Pluronic (with and without B. subtilis) exhibited a 6 -fold increase. This trend changed on day 4, when all groups presented a two-fold increase compared with the untreated group. Post-challenge, on day $8, B a$ cillus counts for the two groups that received B. subtilis increased 4-fold, while counts for the group that received plain Pluronic decreased to the control group level. From day 11, i.e. 4 days after the last administration, control levels were attained for all treatment groups with insignificant differences compared with the untreated group. 


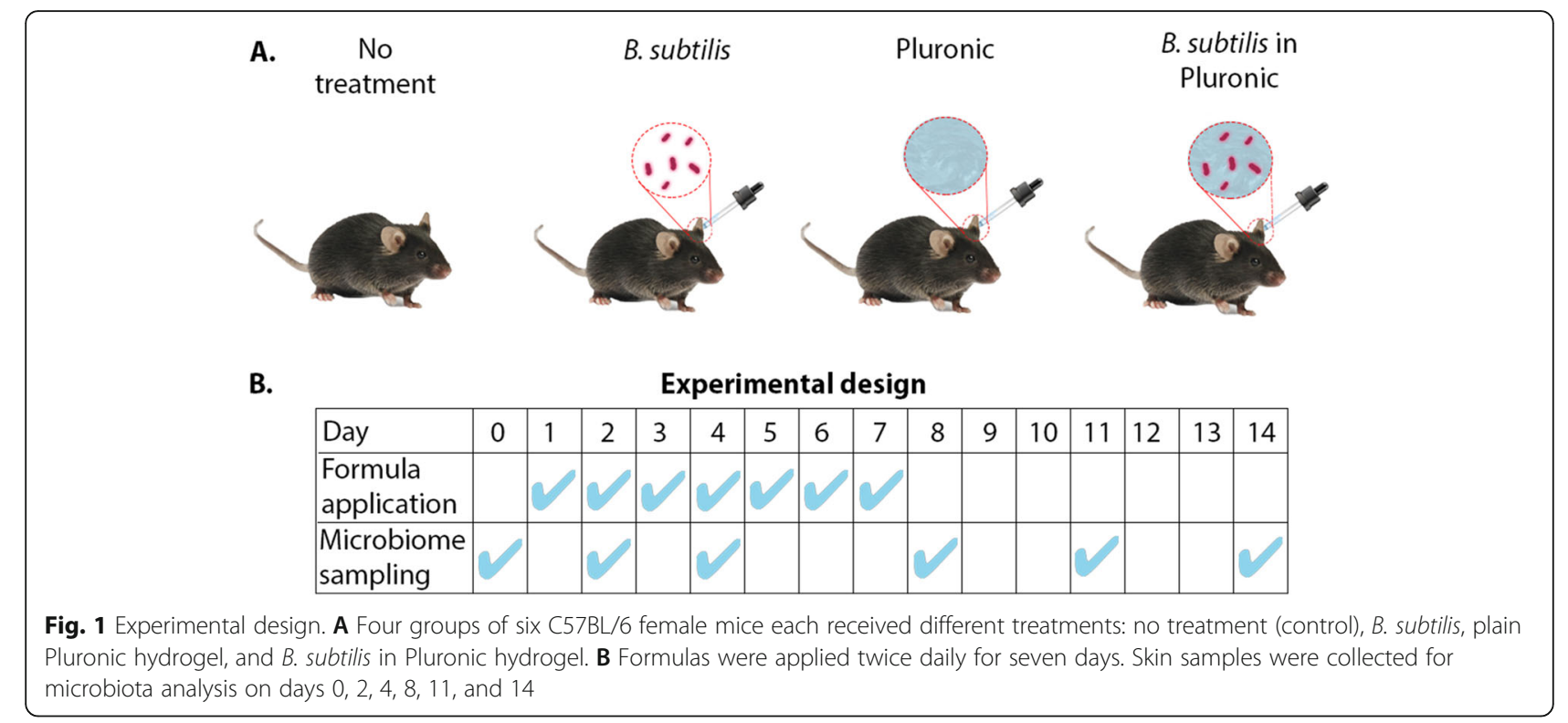

PCA was then used to assess skin microbiota dynamics on both the genus (Fig. 3A) and the species (Fig. 3B and Supplementary Fig. 1) levels. Our data indicated a clear clustering according to treatment day and nature as explained by $\mathrm{PC} 1$ and $\mathrm{PC} 2$, respectively (i.e., data organization according to treatment nature along $\mathrm{PC} 2$ axis can be observed in Fig. 3B). Treatment nature and time point influenced the observed clustering to the same extent, as evidenced by the similarity in effect size between PC1 and PC2 (6.97-6.96\% and 5.57-5.86\%, respectively). The B. subtilis in Pluronic group exhibited an enhanced microbial shift compared with the pure $B$. subtilis group, which showed a very similar pattern but with a slight delay, prominent on day 8 (Fig. 3A). One week after ceasing treatment administration, however, all groups presented a microbiota composition similar to that of the untreated control group.

We further investigated the microbiota shift upon $B$. subtilis intervention and after its cessation by analyzing the intervention's effects on the relative abundance of the most represented skin bacterial genera (Fig. 4A and Supplementary Table 2). Consistent with the PCA results, altered bacterial abundance was observed for all treatment groups along the experiment. For Corynebacterium, the most abundant genera in the ear skin microbiota, application of B. subtilis in Pluronic resulted in a sharp increase from day 2 to 4 , followed by a plateau for the remainder of the experiment. Application of pure $B$. subtilis caused an abundance increase only on day 8 , which remained until day 11 and then decreased to
A.

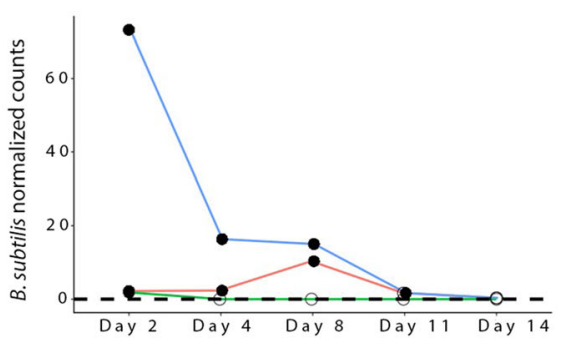

B. subtilis in pluronic

- Pluronic

- B.subtilis

- - No treatment
B.

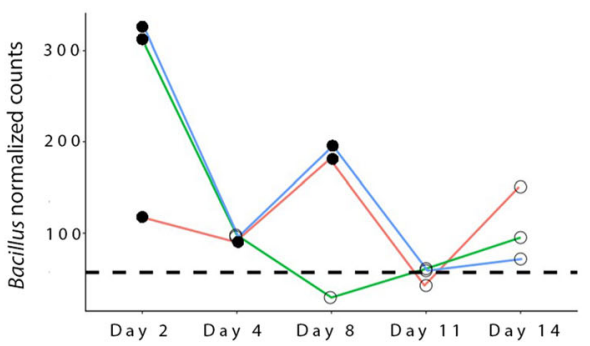

O Non-significant

- Significant

Fig. 2 Temporal and treatment-dependent alteration of Bacillus subtilis species (A) and Bacillus genus (B). Dashed line represents average counts of control samples. Bacillus and B. subtilis counts that differ statistically significantly from control (padj $<0.05$, Wald-test) are denoted by solid circles, while empty circles represent lack of significant difference 

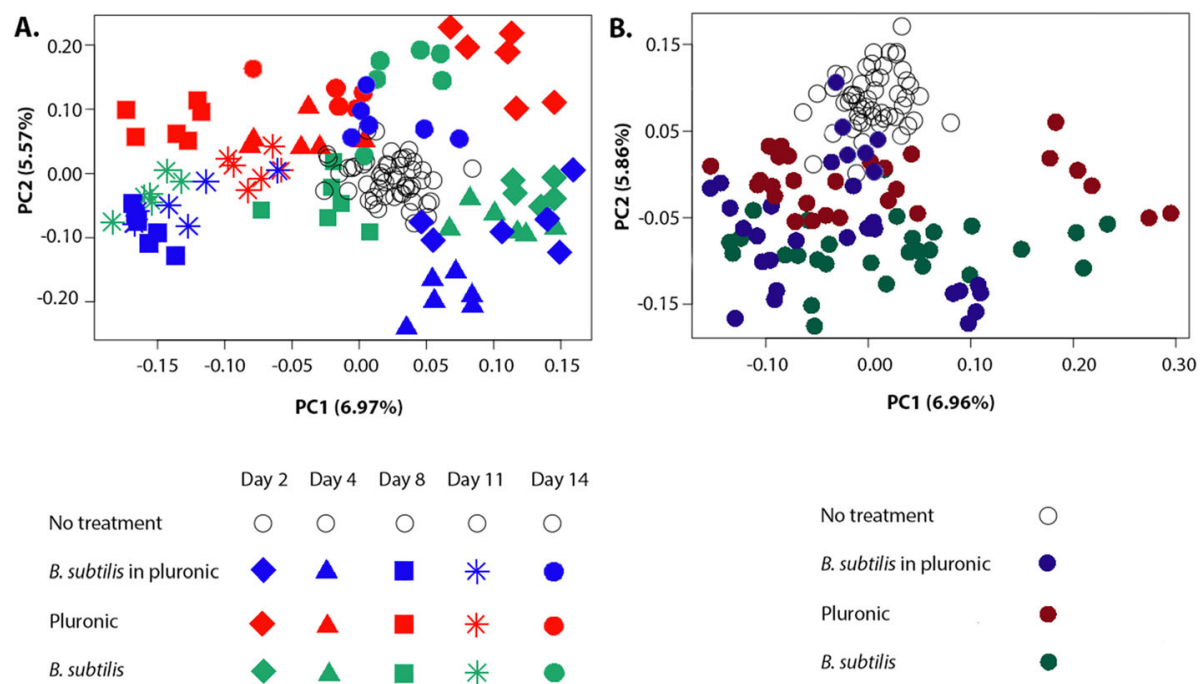

Fig. 3 Principal components analysis (PCA) of skin bacterial communities at the genus (A) and species (B) levels. Axes explain the effect of timing (PC1) and treatment (PC2) on the observed changes

initial values. No significant variations in abundance were observed following the application of pure Pluronic. Interestingly, the relative abundance of Staphylococcus exhibited an inverse trend compared with Bacillus for both B. subtilis-containing formulations: when Bacillus abundance increased, Staphylococcus counts decreased, and vice versa (Spearman rank correlation rho $=-0.6769414, p=4.5^{*} 10^{-13}$ ). To obtain a broader view of these bacterial changes, we mapped the differential representation of bacterial genera along the treatment period (Fig. 4B). Only statistically significant differences in bacterial abundance $(-1<\log 2 \mathrm{FC}>1$; padj $<0.05$, Wald-test) were considered for the analysis (Supplementary Table 3). Following the administration of B. subtilis in Pluronic, several highly related genera were observed to cluster, being either underrepresented or overrepresented compared with the untreated control group (Fig. 4B). For instance, the Lentibacillus, Gemella, Marinococcus, and Virgibacillus genera, of the Bacilliales order, were overrepresented on day 4. The Bacillus genus, on the other hand, was overrepresented on days 2 and 8 , while no statistically significant difference to the control was presented on day 4, consistent with the trend observed in the PCA (Fig. 2B). Staphylococcus abundance decreased during the application of $B$. subtilis in Pluronic formulation (days 2 and 4) and increased on day 11 , three days after the last administration.

\section{Discussion}

The concept of skin microbiota manipulation, either by promoting bacterial balance or by pathogen inhibition, is well established [35, 36]. However, despite significant progress in the field, transplantation of bacteria or bacterial ingredients that selectively stimulate or inhibit the growth and activity of one or a limited number of bacterial species is still at a very experimental stage in skin therapy [4]. Local delivery of live bacterial therapies is often challenging. Live bacterial therapy is often challenging as a local delivery system since the bacteria must reach the site of action alive and establish themselves there [15], hence the importance of proper formulation design. Moreover, the effect of the live-bacteria formulation observed here was limited to treatment course. Skin microorganisms play an essential role in maintaining many aspects of human health including protection against pathogens, education of our immune system, and the breakdown of natural products [2]. Since these aspects of skin microbiota are not fully understood, a transient, temporary effect may be viewed as an advantageous over a stable, long-term modulation.

In this study, administration of B. subtilis to the ear skin of mice resulted in the modulation of the skin microbial composition. Our results indicate that a suitable dermal delivery system is of prime importance for successful administration of live bacteria and, consequently, for microbiota modulation. Administration of B. subtilis in Pluronic hydrogel resulted in a significant increase in B. subtilis counts compared with a more moderate increase in bacterial levels in the absence of Pluronic. Significant differences $(\mathrm{p}<0.05)$ was found between $B$. subtilis in Pluronic and B. subtilis groups on days 2 and 4 but not on day 8 . The ability of B. subtilis to support the growth of other members of the genus Bacillus was explained by cell-cell interactions that induce biofilm production and by the immunity of bacillus to molecules 
A.

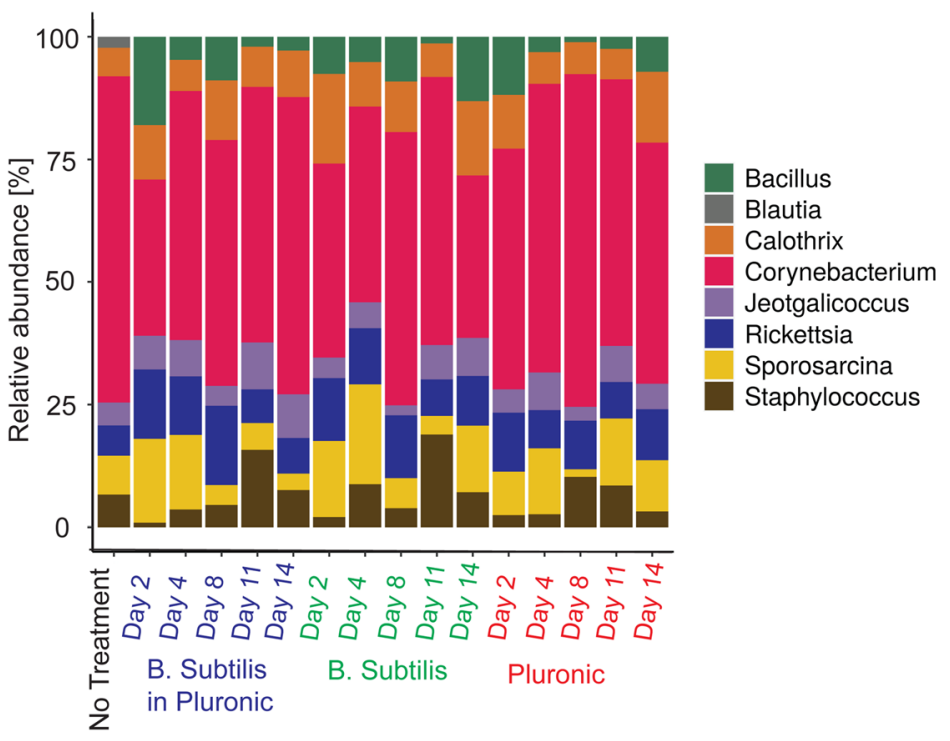

B.

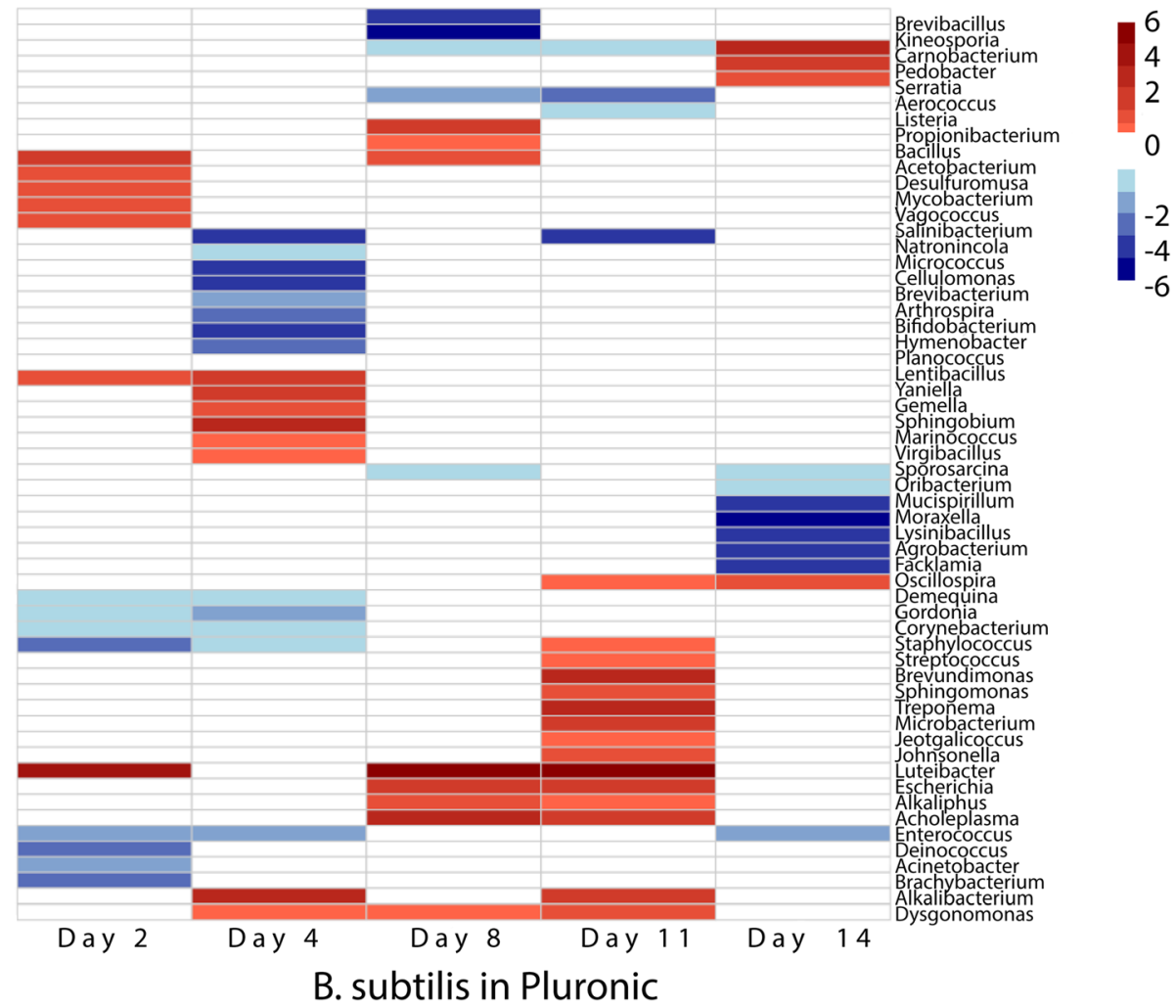

Fig. 4 Effect of $B$. subtilis challenge on the microbial composition of the skin. A Relative abundance plot for the most represented bacterial genera in the ear skin microbiota. B Differential representation of bacterial genera along treatment with B. subtilis in Pluronic hydrogel compared with the untreated control group (B). Statistically significant differences in bacterial abundance were considered $(-1<\log 2 \mathrm{FC}>1$; padj $<0.05$, Wald-test)

secreted from closely related bacteria [37]. The effect of Pluronic hydrogel on the Bacillus genera was significant during the first two days of application: the two Pluronic groups (pure Pluronic and B. subtilis in Pluronic) showed a fast increase in Bacillus abundance that surpassed the effect exhibited by the pure B. subtilis group. The enhanced performance of the $B$. subtilis in Pluronic formulation can be explained by the potential contribution of various factors. One is the ability of Pluronic F-127 to selectively reduce the attachment and 
biofilm formation of several bacteria [38], probably owing to its surfactant properties [39]. Another explanation is that Pluronic F-127 gel serves as a protective layer between the bacteria and the skin, aiding the delivery of B. subtilis to the desired site. Pluronic F-127 was found to be very effective in reducing the degradation of such peptides while sustaining their delivery from the hydrogel to its surroundings [40, 41]. Finally, the ability of Pluronic gel to enhance the immune response, probably by stimulating the expression of vascular epithelial growth factors, may be related to the selective shift in the skin microbial composition [42]. We note that the differences in microbiome composition can also be attributed to specific displacement of certain microorganisms or to an overall decrease in microbiota abundance. Nevertheless, the present study is, to our knowledge, among the first to evaluate the use of biomaterials in live bacterial skin delivery systems for microbiota modulation. The application of $B$. subtilis altered the abundance of common skin bacteria, including Staphylococcus and Corynebacterium spp. Some of these bacterial genera have been reported to be the most abundant organisms colonizing moist areas of the skin, including the antecubital fossa in humans, which the ear skin of mice resembles [43, 44]. Common skin commensals belonging to the Corynebacterium genus have been shown to exhibit antimicrobial activity against human pathogens and to stimulate healthy host-bacterial interactions [45]. For example, C. striatum can influence $S$. aureus gene expression by downregulating virulence-related genes and upregulating genes associated with the establishment of a commensal relationship [46].

\section{Conclusions}

In this study, we investigated the effect of challenging the skin microbiota with $B$. subtilis, a bacterium with therapeutic potential [24, 25, 27]. The carrier, a Pluronic F-127 hydrogel, was found to facilitate B. subtilis administration and enhance its activity, possibly by supporting continuous bacterial growth and providing better conditions for skin attachment. The interaction between the polymer, B. subtilis, and epithelial cells should be further investigated to provide more insight into the mechanism of action. We demonstrated that $B$. subtilis induces a shift in the skin microbiota composition that is facilitated by the presence of the live bacteria in Pluronic hydrogel. This alteration was characterized by a shift in the skin microbiota, influencing the abundance of commensal, beneficial and pathogenic bacteria. Given the great potential of the live bacterial delivery approach, its clinical value for the treatment of conditions associated with skin microbiota dysbiosis (i.e. atopic dermatitis or acne) should be investigated.

\section{Methods}

\section{Animal husbandry}

Twenty-four 8-week old C57BL/6 female mice were purchased from Envigo, Israel. We restricted the experiment to female mice in order to avoid any possible interference of sex factors with the variability of microbial flora. The animals were caged randomly in four groups of six mice each. All animals were maintained in sterilized cages on a 12-h light/12-h dark cycle with food and water provided ad libitum. Bedding was changed once a week, and mice were given an autoclaved chow diet and sterilized water. Moderate dermal irritation or $20 \%$ body weight loss were used to determine the humane endpoints. At the end of the study, mice were sacrificed by $\mathrm{CO}_{2}$ asphyxiation following protocols approved by the corresponding authority.

\section{Formulations and administration}

Each independently housed group of six mice received a different treatment formulation twice daily, every $12 \mathrm{~h}$, for 7 days. B. subtilis 3610 was chosen since it is a natural, non-modified wild type strain. The treatment formulations consisted of (a) $10 \% \mathrm{v} / \mathrm{v}$ B. subtillis in lysogeny broth (LB) with $18 \%$ w/v Pluronic ("B. subtilis formulation"), (b) $18 \% \mathrm{w} / \mathrm{v}$ Pluronic, (c) $10 \% \mathrm{v} / \mathrm{v}$ B. subtillis in LB, and (d) untreated control. A stock of Pluronic F-127 was prepared by dissolving the appropriate amount of polymer in distilled water to obtain a final concentration of $20 \% \mathrm{w} / \mathrm{v}$. A stock solution of B. subtilis was cultured in LB agar and incubated at $37{ }^{\circ} \mathrm{C}$ overnight after which a bacterial colony was transferred to a falcon tube containing fresh liquid LB, incubated at $37{ }^{\circ} \mathrm{C}$ and allowed to reach an optical density (OD) of 0.6 at $600 \mathrm{~nm}$. The B. subtilis formulation was prepared by adding $0.5 \mathrm{~mL}$ of fresh bacterial culture $\left(\mathrm{OD}_{600}\right.$ : 0.6$)$ to $4.5 \mathrm{~mL}$ of $20 \% \mathrm{w} / \mathrm{v}$ Pluronic, obtaining a final solution of $18 \% \mathrm{w} / \mathrm{v}$ Pluronic with $10 \% \mathrm{v} / \mathrm{v}$ B. subtilis in LB. $18 \% \mathrm{w} / \mathrm{v}$ Pluronic was obtained by adding $0.5 \mathrm{~mL}$ of liquid LB to $4.5 \mathrm{~mL}$ of Pluronic $20 \% \mathrm{w} / \mathrm{v}$. To prepare $10 \% \mathrm{v} / \mathrm{v} \mathrm{B}$. subtillis in LB, $0.5 \mathrm{~mL}$ of fresh bacterial culture $\left(\mathrm{OD}_{600}\right.$ : 0.6$)$ were added to $4.5 \mathrm{~mL}$ of fresh $\mathrm{LB}$ media. The different formulations $(100 \mu \mathrm{L})$ were administered to both left and right ears of the animals of the corresponding groups. Pluronic-containing formulations were allowed to harden on the skin for $1 \mathrm{~min}$ until a viscous gel was obtained.

\section{Sample collection}

To sample the skin microbiota, both ears were thoroughly swabbed with a sterile FLOQSwab presoaked in buffer solution $(0.15 \mathrm{M} \mathrm{NaCl}$ and $0.1 \%$ Tween 20$)$. Sampling was effectuated every other day from day 0 , before formulation administration, to day 14, a week after last 
application. Samples were stored at $-80{ }^{\circ} \mathrm{C}$ until processing.

\section{Bacterial DNA extraction}

DNA extraction and purification were performed using the PureLink Microbiome Kit (Invitrogen, Thermo Fisher Scientific) according to manufacturer's protocol and supplementary instructions for low bio-burden samples. Concentration of purified DNA was determined using the QuBit High Sensitivity DNA quantification system (Invitrogen) and stored at $20{ }^{\circ} \mathrm{C}$ until further use.

\section{S rRNA gene amplification and sequencing}

$16 \mathrm{~S}$ rRNA gene amplification and sequencing were carried out at the Technion's Genome Center. Sequencing libraries were prepared using the $16 \mathrm{~S}$ rRNA Metagenomic Sequencing Library Preparation protocol by Illumina with minor adjustments. Sample input was $0.625 \mathrm{ng}$ of genomic DNA and the first PCR amplification consisted of 30 cycles. V3 and V4 hypervariable regions of bacterial $16 \mathrm{~S}$ rRNA were the amplification targets. Primers used to target the V3 and V4 regions of the $16 \mathrm{~S}$ rRNA gene were 5'TCGTCGGCAGCGTCAG ATGTGTATAAGAGACAGCCTACGGGNGGCWGCAG (Forward) and 5'GTCTCGTGGGCTCGGAGATGTGTA TAAGAGACAGGACTACHVGGGTATCTAATCC (Reverse). The respective Illumina overhang adapter sequences were included in the primer design. All 96 libraries were sequenced on an Illumina MiSeq instrument with 250 pairedends reads. Sequencing data was input into the $16 \mathrm{~S}$ rRNA gene Metagenomics app on the Illumina BaseSpace sequence hub. Classification was performed using the Illumina $16 \mathrm{~S}$ rRNA gene Metagenomics workflow, which includes demultiplexing of indexed reads, FASTQ files generation, and read classification. Operational Taxonomic Unit (OTU) clustering and classification were performed at genus and species level. An Illumina-curated version of the Greengenes database was used as the taxonomy database for the metagenomics workflow. The algorithm used is a high-performance implementation of the Ribosomal Database Project Classifier described in Wang Q. et al. [47].

\section{Bioinformatics analyses}

The R statistical software package was used for all statistical tests. The Remove Unwanted Variation (RUV) normalization strategy described by Risso et al. [34] was employed to remove noise from unknown sources. Factors of unwanted variation using replicate samples were estimated using the RUVseq package. Parameters were set to $K=15$ at the species level and $K=40$ at the genus level, and corrected counts were calculated. For the calculations, the no-treatment control treatment was defined as all no-treatment samples and all day-0 samples (before treatment). Principal components analysis (PCA) was applied on RUVseq corrected counts with Euclidean distance as similarity metric using EDAseq [48]. A design matrix including both the covariates of interest and the factors of unwanted variation was supplied to DESeq2 for differential analysis [49]. To test the effect of treatments, each treatment was compared with the no treatment control using Wald test and the False Discovery Rate correction for multiple comparisons as implemented in DESeq2 [49]. Only bacteria with at least 50 counts in 2 or more samples were included in the analysis. A bacterial genus or species was considered to differ significantly between treatments if the absolute value fold change was at least 2 , with an adjusted $p$-value (padj) smaller than 0.05 .

\section{Abbreviations \\ LB: Lysogeny broth; OD: Optical density; OTU: Operational taxonomic unit; PCA: Principal component analysis; PCR: Polymerase chain reaction; RUV: Remove unwanted variation}

\section{Supplementary Information}

The online version contains supplementary material available at https:/doi. org/10.1186/s12866-021-02295-y

Additional file 1: Supplementary Figure 1. Principal coordinates analysis (PCA) of skin bacterial communities at species level. Each treatment group and treatment day is distinguished by a different color. Empty dots represent control samples.

\section{Additional file 2.}

Additional file 3: Supplementary Table 2. Relative abundance of the seven most represented bacterial genera for different treatments ( $B$. subtilis in Pluronic, B. subtilis and Pluronic) and treatment days $(2,4,8,11$ and 14). Each experimental group consisted of 6 mice, named A-F for replicates. For each bacterial genus, given a treatment and treatment day, the mean and relative abundance in percentage (Rel. Ab. (\%)) are calculated.

Additional file 4: Supplementary Table 3. Differential bacterial species resulting from $B$. subtilis in Pluronic treatment on different treatment time points, identified by DESeq2 $(-1<\log 2$ FC $>1$; padj $<0.05$, Wald-test). Only bacteria with at least 50 counts in two or more samples were included in the analysis.

\section{Acknowledgements}

We are grateful to Dr. Adi Gross for helping in the laboratory. We thank Prof. Ravid Sraussman and Dr. Shiri Meshner for their kind advices in sample collection.

\section{Authors' contributions}

VM and BM conceived the study. VM carried out laboratory work with help from RB. VM carried out the in-vivo work with help from animal facility staff. VM analyzed the data with help from RB and GH. VM wrote the paper with the help from all co-authors. All authors read and approved the final manuscript.

\section{Funding}

This work was financially supported by the Israeli Science Foundation (grants no. 515/20). The funding source provided funds for purchase of consumption materials for the study and for the animal facility, but had no role in the design of the study and collection, analysis or writing of the manuscript.

\section{Availability of data and materials}

The datasets generated and/or analysed during the current study are available in the BioProject database repository (BioProject ID PRJNA640303): https://www.ncbi.nlm.nih.gov/bioproject/640303. Original R scripts are available in GitHub under request. 


\section{Declarations}

\section{Ethics approval and consent to participate}

All animals' experimental procedures used in this study were approved by the Animal Policy and Welfare Committee, approval number IL-025-02-2019, Technion, Israel Institute of Technology and were in conformity with the guideline of the Israel Council on Animal Care.

\section{Consent for publication}

Not applicable.

\section{Competing interests}

The authors declare that they have no competing interests.

\section{Author details}

${ }^{1}$ Faculty of Biotechnology and Food Engineering, Technion - Israel Institute of Technology, 3200003 Haifa, Israel. Bioinformatics Knowledge Unit, The Lorry I. Lokey Interdisciplinary Center for Life Sciences and Engineering, Technion - Israel Institute of Technology, 3200003 Haifa, Israel. ${ }^{3}$ Faculty of Biology, Technion - Israel Institute of Technology, 3200003 Haifa, Israel.

\section{Received: 15 July 2020 Accepted: 12 August 2021}

\section{Published online: 21 August 2021}

\section{References}

1. Guarner F, Bourdet-Sicard R, Brandtzaeg P, Gill HS, McGuirk P, Van Eden W, Versalovic J, Weinstock JV, Rook GA. Mechanisms of disease: the hygiene hypothesis revisited. Nat Rev Gastroenterol Hepatol. 2006;3:275.

2. Byrd AL, Belkaid Y, Segre JA. The human skin microbiome. Nat Rev Microbiol. 2018;16:143.

3. Prescott SL, Larcombe D-L, Logan AC, West C, Burks W, Caraballo L, Levin M, Van Etten $E$, Horwitz P, Kozyrskyj A. The skin microbiome: impact of modern environments on skin ecology, barrier integrity, and systemic immune programming. World Allergy Org J. 2017;10:29

4. Levy M, Kolodziejczyk AA, Thaiss CA, Elinav E. Dysbiosis and the immune system. Nat Rev Immunol. 2017;17:219.

5. Relman DA. The human microbiome: ecosystem resilience and health. Nutr Rev. 2012;70:2-9.

6. Petersen C, Round JL. Defining dysbiosis and its influence on host immunity and disease. Cell Microbiol. 2014;16:1024-33.

7. Schommer NN, Gallo RL. Structure and function of the human skin microbiome. Trends Microbiol. 2013;21:660-8.

8. Kong HH, Oh J, Deming C, Conlan S, Grice EA, Beatson MA, Nomicos E, Polley EC, Komarow HD, Murray PR. Temporal shifts in the skin microbiome associated with disease flares and treatment in children with atopic dermatitis. Genome Res. 2012:22:850-9.

9. Fitz-Gibbon S, Tomida S, Chiu B-H, Nguyen L, Du C, Liu M, Elashoff D, Erfe MC, Loncaric A, Kim J. Propionibacterium acnes strain populations in the human skin microbiome associated with acne. J Invest Dermatol. 2013;133: 2152-60.

10. Ganju P, Nagpal S, Mohammed M, Kumar PN, Pandey R, Natarajan VT, Mande SS, Gokhale RS. Microbial community profiling shows dysbiosis in the lesional skin of Vitiligo subjects. Sci Rep. 2016;6:18761.

11. Lee HJ, Jeong SE, Lee S, Kim S, Han H, Jeon CO. Effects of cosmetics on the skin microbiome of facial cheeks with different hydration levels. Microbiologyopen. 2018;7:e00557

12. Chien AL, Tsai J, Leung S, Mongodin EF, Nelson AM, Kang S, Garza LA. Association of systemic antibiotic treatment of acne with skin microbiota characteristics. JAMA Dermatol. 2019:155:425-34.

13. Dellacecca ER, Cosgrove C, Mukhatayev Z, Akhtar S, Engelhard VH, Rademaker AW, Knight K, Le Poole IC. Antibiotics drive microbial imbalance and vitiligo development in mice. J Invest Dermatol. 2019;140(3):676-87.

14. Maguire M, Maguire $\mathrm{G}$. The role of microbiota, and probiotics and prebiotics in skin health. Arch Dermatol Res. 2017:309:411-21.

15. Moskovicz V, Gross A, Mizrahi B. Extrinsic factors shaping the skin microbiome. Microorganisms. 2020;8(7):1023.

16. Dethlefsen L, Relman DA. Incomplete recovery and individualized responses of the human distal gut microbiota to repeated antibiotic perturbation. Proc Natl Acad Sci. 2011;108:4554-61.

17. Watanabe J, Fujiwara R, Sasajima N, Ito S, Sonoyama K. Administration of antibiotics during infancy promoted the development of atopic dermatitis- like skin lesions in NC/Nga mice. Biosci Biotechnol Biochem. 2010;74(2):35863.

18. Willing BP, Russell SL, Finlay BB. Shifting the balance: antibiotic effects on host-microbiota mutualism. Nat Rev Microbiol. 2011:9:233.

19. Zhang M, Jiang Z, Li D, Jiang D, Wu Y, Ren H, Peng H, Lai Y. Oral antibiotic treatment induces skin microbiota dysbiosis and influences wound healing. Microbial Ecol. 2015:69:415-21.

20. Nakatsuji T, Chen TH, Narala S, Chun KA, Two AM, Yun T, Shafiq F, Kotol PF, Bouslimani A, Melnik AV. Antimicrobials from human skin commensal bacteria protect against Staphylococcus aureus and are deficient in atopic dermatitis. Sci Transl Med. 2017:9:eaah4680.

21. Myles IA, Earland NJ, Anderson ED, Moore IN, Kieh MD, Williams KW, Saleem A, Fontecilla NM, Welch PA, Darnell DA. First-in-human topical microbiome transplantation with Roseomonas mucosa for atopic dermatitis. JCI Insight 2018;3:e120608

22. Paetzold B, Willis JR, de Lima JP, Knödlseder N, Brüggemann H, Quist SR, Gabaldón T, Güell M. Skin microbiome modulation induced by probiotic solutions. Microbiome. 2019;7:95

23. Lee GR, Maarouf M, Hendricks AJ, Lee DE, Shi VY. Topical probiotics: the unknowns behind their rising popularity. Dermatol Online J. 2019;25:13030

24. Earl AM, Losick R, Kolter R. Ecology and genomics of Bacillus subtilis. Trends Microbiol. 2008;16:269-75.

25. Gonzalez DJ, Haste NM, Hollands A, Fleming TC, Hamby M, Pogliano K, Nizet V, Dorrestein PC. Microbial competition between Bacillus subtilis and Staphylococcus aureus monitored by imaging mass spectrometry. Microbiology. 2011;157:2485

26. Dekio I, Hayashi H, Sakamoto M, Kitahara M, Nishikawa T, Suematsu M, Benno Y. Detection of potentially novel bacterial components of the human skin microbiota using culture-independent molecular profiling. J Med Microbiol. 2005:54:1231-8.

27. Lee N-K, Kim W-S, Paik H-D. Bacillus strains as human probiotics: characterization, safety, microbiome, and probiotic carrier. Food Sci Biotechnol. 2019;28:1297-305.

28. Lufton M, Bustan O, Eylon B-h, Shtifman-Segal E, Croitoru-Sadger T, Shagan A, Shabtay-Orbach A, Corem-Salkmon E, Berman J, Nyska A, Mizrahi B. Living bacteria in thermoresponsive gel for treating fungal infections. Adv Func Mater. 2018;28:1801581.

29. Coyte KZ, Schluter J, Foster KR. The ecology of the microbiome: networks, competition, and stability. Science. 2015;350:663-6.

30. Oh J, Byrd AL, Park M, Kong HH, Segre JA, Program NCS. Temporal stability of the human skin microbiome. Cell. 2016;165:854-66.

31. Kong HH, Segre JA. Skin microbiome: looking back to move forward. J Invest Dermatol. 2012;132:933-9.

32. Fritz JV, Desai MS, Shah P, Schneider JG, Wilmes P. From meta-omics to causality: experimental models for human microbiome research. Microbiome. 2013;1:14

33. Grice EA, Kong HH, Renaud G, Young AC, Bouffard GG, Blakesley RW, Wolfsberg TG, Turner ML, Segre JA, Program NCS. A diversity profile of the human skin microbiota. Genome Res. 2008:18(7):1043-50.

34. Risso D, Ngai J, Speed TP, Dudoit S. Normalization of RNA-seq data using factor analysis of control genes or samples. Nature biotechnology. 2014;32:896.

35. Lolou V, Panayiotidis MI. Functional role of probiotics and prebiotics on skin health and disease. Fermentation. 2019:5:41.

36. Knackstedt R, Knackstedt T, Gatherwright J. The role of topical probiotics on skin conditions: a systematic review of animal and human studies and implications for future therapies. Exp Dermatol. 2019;29(1): $15-21$.

37. Shank EA, Klepac-Ceraj V, Collado-Torres L, Powers GE, Losick R, Kolter R. Interspecies interactions that result in $<$ em $>$ Bacillus subtilis forming biofilms are mediated mainly by members of its own genus. Proc Natl Acad Sci. 2011;108:E1236-43.

38. Alvarado-Gomez E, Martínez-Castañon G, Sanchez-Sanchez R, GanemRondero A, Yacaman MJ, Martinez-Gutierrez F. Evaluation of anti-biofilm and cytotoxic effect of a gel formulation with Pluronic F-127 and silver nanoparticles as a potential treatment for skin wounds. Mater Sci Eng C. 2018;92:621-30.

39. Van Hamme JD, Singh A, Ward OP. Physiological aspects: part 1 in a series of papers devoted to surfactants in microbiology and biotechnology. Biotechnol Adv. 2006;24:604-20.

40. Wenzel JGW, Balaji KSS, Koushik K, Navarre C, Duran SH, Rahe CH, Kompella UB. Pluronic ${ }^{\circledR}$ F127 gel formulations of Deslorelin and $\mathrm{GnRH}$ reduce drug 
degradation and sustain drug release and effect in cattle. J Controlled Release. 2002;85:51-9.

41. Kohane DS, Langer R. Drug delivery and translation. Drug Deliv Transl Res. 2011;1:4-6.

42. Kant V, Gopal A, Kumar D, Gopalkrishnan A, Pathak NN, Kurade NP, Tandan SK, Kumar D. Topical pluronic F-127 gel application enhances cutaneous wound healing in rats. Acta Histochem. 2014;116:5-13.

43. Grice $E A$, Kong $H H$, Conlan $S$, Deming CB, Davis J, Young AC, Bouffard GG, Blakesley RW, Murray PR, Green ED. Topographical and temporal diversity of the human skin microbiome. Science. 2009;324:1190-2.

44. SanMiguel A, Grice EA. Interactions between host factors and the skin microbiome. Cell Mol Life Sci. 2015;72:1499-515.

45. Chen YE, Fischbach MA, Belkaid Y. Skin microbiota-host interactions. Nature. 2018;553:427-36.

46. Ramsey MM, Freire MO, Gabrilska RA, Rumbaugh KP, Lemon KP. Staphylococcus aureus shifts toward commensalism in response to Corynebacterium species. Front Microbiol. 2016;7:1230.

47. Wang Q, Garrity GM, Tiedje JM, Cole JR. Naive Bayesian classifier for rapid assignment of rRNA sequences into the new bacterial taxonomy. Appl Environ Microbiol. 2007;73:5261-7.

48. Risso D, Schwartz K, Sherlock G, Dudoit S. GC-content normalization for RNA-Seq data. BMC Bioinform. 2011:12:480.

49. Love Ml, Huber W, Anders S. Moderated estimation of fold change and dispersion for RNA-seq data with DESeq2. Genome Biol. 2014;15:550.

\section{Publisher's Note}

Springer Nature remains neutral with regard to jurisdictional claims in published maps and institutional affiliations.

Ready to submit your research? Choose BMC and benefit from:

- fast, convenient online submission

- thorough peer review by experienced researchers in your field

- rapid publication on acceptance

- support for research data, including large and complex data types

- gold Open Access which fosters wider collaboration and increased citations

- maximum visibility for your research: over $100 \mathrm{M}$ website views per year

At $\mathrm{BMC}$, research is always in progress.

Learn more biomedcentral.com/submissions 